\title{
Influence comparée d'une alimentation à base de graines de soja cuites ou grillées sur les performances des porcs en croissance finition
}

\author{
F. Meffeja ${ }^{1}$ T. Dongmo ${ }^{1}$ N. Njifutie ${ }^{2}$ \\ J. Djoukam ${ }^{3}$ J.M. Fotso ${ }^{1}$
}

\author{
Mots-clés \\ Porcin - Porcelet - Tourteau de soja - \\ Graine - Cuisson - Grillage - \\ Croissance - Cameroun.
}

\begin{abstract}
Résumé
Une expérience a été menée pour évaluer l'efficacité des traitements thermiques par grillade (chaleur sèche) et par cuisson (chaleur humide) des graines de soja sur les performances zootechniques des porcs en croissance finition. Vingt porcelets hybrides mâles, de poids moyen initial de 15,4 $\pm 0,8 \mathrm{~kg}$, ont été répartis en quatre lots (cinq répétitions par lot) et soumis à des rations alimentaires à base des tourteaux de soja, de coton et de graines de soja cuites ou grillées pendant une période de 98 jours. Les rations à base de tourteaux de soja et de coton couramment utilisées ont servi de rations témoins. Les résultats ont montré que le gain pondéral et l'indice de consommation obtenus à partir des rations des graines cuites et grillées ont été significativement supérieurs $(p<0,05)$ à ceux de la ration du tourteau de coton, mais ils n'ont pas montré de différence significative $(p>0,05)$ par rapport à la ration du tourteau de soja. Une comparaison des deux méthodes de traitements n'a présenté aucune différence significative sur les performances zootechniques des animaux. Les coûts alimentaires de production d'un kilogramme de poids vif, pondérés sur les deux phases de croissance - bien qu'ils n'aient pas été significativement différents - ont montré une légère baisse numérique pour le traitement par grillade. Ceci permet d'affirmer que cette méthode de traitement peut être utilisée aussi avantageusement que le traitement par la chaleur humide.
\end{abstract}

\section{INTRODUCTION}

Une des contraintes majeures au développement de la production porcine au Cameroun demeure l'approvisionnement irrégulier et le coût élevé des concentrés protéiques entrant dans l'alimentation animale. Le tourteau de soja, qui est importé, reste encore l'unique source de protéine équilibrée utilisée par les provenderies locales. Il est devenu une source de protéines très coûteuse et difficile d'accès à cause de sa forte demande sur les marchés occidentaux (8).

\footnotetext{
1. Centre régional de recherche agricole pour le développement de Nkolbisson, S/C BP 7070, Yaoundé, Cameroun

E-mail : meffeja@yahoo.fr

2. Université de Yaoundé I, département de biologie et physiologie animale

3. Université de Dschang, faculté des Sciences agronomiques
}

Les graines de soja et d'autres graines de légumineuses tropicales dont la culture est vulgarisée dans beaucoup de pays africains (11) pourraient constituer une source alternative de protéines et d'énergie, indispensables aux élevages porcins et de volailles, et réduire ainsi d'importantes ressources financières actuellement investies dans l'importation des concentrés protéiques.

Les méthodes de traitement appliquées aux graines de soja en vue de réduire les facteurs antinutritionnels $(7,12)$ ont montré que l'activité de la plupart de ces facteurs est détruite uniquement par la chaleur humide (cuisson). Dès lors, les travaux publiés sur leur utilisation chez les monogastriques portent en majorité sur le traitement par la chaleur humide $(1,4,6,14)$. Les recherches sur leur utilisation sous forme grillée sont rares. L'objectif de la présente étude a été de comparer l'efficacité des traitements thermiques des graines de soja par grillade et par cuisson sur les performances des porcs en croissance finition. 


\section{MATERIEL ET METHODES}

Vingt porcelets mâles croisés Landrace $\mathrm{x}$ Duroc $\mathrm{x}$ Large White $\mathrm{x}$ Berkshire, de poids moyen initial $15,4 \pm 0,8 \mathrm{~kg}$, ont été répartis dans 20 loges expérimentales en bois, au sol cimenté ( $2 \mathrm{~m}$ x 2,5 m) et soumis à quatre rations (cinq répétitions par traitement) formulées à base du tourteau de soja (TS), de coton (TC) et de graines de soja cuites (SC) ou grillées (SG), de manière à fournir 21 p. 100 de protéines brutes en phase de croissance (tableau I) et 18 p. 100 en phase de finition. (tableau II). Les rations à base des tourteaux de soja et de coton couramment utilisées ont servi de rations témoins. Les animaux ont été nourris une fois par jour et les refus enregistrés le jour suivant pendant une période de 98 jours. Ils ont été pesés au début de l'expérience et tous les 15 jours à jeun à l'aide d'une bascule Marshall type PM 100, de portée $200 \pm 0,1 \mathrm{~kg}$.

Les graines de soja utilisées ont été divisées en deux parties : la première partie a été cuite au feu de bois dans une marmite par tranche de $10 \mathrm{~kg}$ à raison de $1,5 \mathrm{l}$ d'eau/kg pendant 40 à $50 \mathrm{~min}$. La deuxième partie a été grillée au feu de bois, dans une marmite

\section{Tableau I}

Composition centésimale des rations expérimentales (phase de croissance)

\begin{tabular}{|c|c|c|c|c|}
\hline Ingrédients & TS & TC & SC & SG \\
\hline Maïs (\%) & 43,5 & 45,5 & 39 & 39 \\
\hline Farine de poisson (\%) & 2 & 2 & 2 & 2 \\
\hline Farine de sang (\%) & 3 & 3 & 3 & 3 \\
\hline Tourteau de soja (\%) & 18 & - & - & - \\
\hline Tourteau de coton (\%) & - & 20 & - & - \\
\hline Soja cuit (\%) & - & - & 22,5 & - \\
\hline Soja grillé (\%) & - & - & - & 22,5 \\
\hline Son de blé (\%) & 12 & 8 & 12 & 12 \\
\hline Tourteau de palmiste (\%) & 18 & 18 & 18 & 18 \\
\hline Poudre d'os (\%) & 3 & 3 & 3 & 3 \\
\hline Sel (\%) & 0,5 & 0,5 & 0,5 & 0,5 \\
\hline Total (\%) & 100 & 100 & 100 & 100 \\
\hline \multicolumn{5}{|c|}{ Composition chimique calculée } \\
\hline $\begin{array}{l}\text { Energie digestible } \\
(\mathrm{kcal} / \mathrm{kg})\end{array}$ & 3086 & 2915,5 & 3248 & 3248 \\
\hline Protéines brutes (\%) & 21,16 & 20,96 & 20,84 & 20,84 \\
\hline Cellulose brute (\%) & 5,21 & 7,09 & 6,1 & 6,1 \\
\hline Matières grasses (\%) & 4,29 & 3 & 6,68 & 6,68 \\
\hline Lysine (\%) & 1,14 & 0,98 & 1,14 & 1,14 \\
\hline Méthionine + cystine $(\%)$ & 0,71 & 0,73 & 0,7 & 0,7 \\
\hline Calcium (\%) & 1,28 & 1,19 & 1,3 & 1,3 \\
\hline Phosphore (\%) & 0,89 & 1,01 & 0,78 & 0,78 \\
\hline \multicolumn{5}{|c|}{ Composition chimique analysée } \\
\hline Matière sèche ( $\%$ du brut) & 89,79 & 89,79 & 89,7 & 90,09 \\
\hline Protéines brutes (\% MS) & 17,13 & 17,4 & 21,81 & 24,87 \\
\hline Matières grasses (\% MS) & 5,83 & 5,01 & 7,91 & 8 \\
\hline Cendres (\% MS) & 7,5 & 6,29 & 6,82 & 7,85 \\
\hline NDF (\% MS) & 44,9 & 40,48 & 48,93 & 49,46 \\
\hline ADF (\% MS) & 19,88 & 20,93 & 26,34 & 23,83 \\
\hline Cellulose brute & 10,55 & 12,56 & 10,34 & 9,83 \\
\hline Coût de la ration $(\mathrm{Fcfa} / \mathrm{kg}$ & 184,2 & 153 & 183,9 & 177 \\
\hline
\end{tabular}

TS : tourteaux de soja

TC : tourteaux de coton

$\mathrm{SC}$ : graines de soja cuites

SG : graines de soja grillées par tranche de $10 \mathrm{~kg}$ pendant 20 à 25 min jusqu'à l'obtention d'une couleur brunâtre des graines, semblable à celle des arachides grillées, dont la pellicule se détache facilement entre les doigts. Les graines de soja brutes et traitées ainsi que les rations alimentaires ont été ensuite analysées au laboratoire de zootechnie de la faculté des Sciences agronomiques de l'université de Dschang suivant les méthodes de l'AOAC (2) et de Goering et Van Soest (6).

\section{Tableau II}

Composition centésimale des rations expérimentales (phase de finition)

\begin{tabular}{|c|c|c|c|c|}
\hline Ingrédients & TS & TC & SC & SG \\
\hline Maïs (\%) & 43,5 & 45 & 39 & 39 \\
\hline Farine de poisson $(\%)$ & 2 & 2,5 & 2 & 2 \\
\hline Tourteau de soja (\%) & 16 & - & - & - \\
\hline Tourteau de coton $(\%)$ & - & 18 & - & - \\
\hline Soja cuit (\%) & - & - & 20 & - \\
\hline Soja grillé (\%) & - & - & - & 20 \\
\hline Son de blé (\%) & 17 & 13 & 17,5 & 17,5 \\
\hline Tourteau de palmiste (\%) & 18 & 18 & 18 & 18 \\
\hline Poudre d'os (\%) & 3 & 3 & 3 & 3 \\
\hline Sel $(\%)$ & 0,5 & 0,5 & 0,5 & 0,5 \\
\hline Total (\%) & 100 & 100 & 100 & 100 \\
\hline \multicolumn{5}{|c|}{ Composition chimique calculée } \\
\hline $\begin{array}{l}\text { Energie digestible } \\
(\mathrm{kcal} / \mathrm{kg})\end{array}$ & 3084 & 2874,5 & \multicolumn{2}{|c|}{$3163,5 \quad 3163,5$} \\
\hline Protéines brutes (\%) & 18,5 & 18,39 & 18,26 & 18,26 \\
\hline Cellulose brute (\%) & 5,67 & 7,33 & 6,5 & 6,5 \\
\hline Matières grasses (\%) & 4,33 & 3,19 & 6,46 & 6,46 \\
\hline Lysine $(\%)$ & 0,9 & 0,75 & 0,9 & 0,9 \\
\hline Méthionine + cystine (\%) & 0,67 & 0,72 & 0,66 & 0,66 \\
\hline Calcium (\%) & 1,17 & 1,2 & 1,17 & 1,17 \\
\hline Phosphore (\%) & 0,92 & 1,03 & 0,92 & 0,92 \\
\hline Coût de la ration $(\mathrm{Fcfa} / \mathrm{kg})$ & उ) 168,1 & 142,6 & 167,3 & 161,2 \\
\hline
\end{tabular}

TS : tourteaux de soja

TC : tourteaux de coton

$\mathrm{SC}:$ graines de soja cuites

SG : graines de soja grillées

\section{Tableau III}

Caractéristiques chimiques analysées et coûts des traitements thermiques des graines de soja

\begin{tabular}{lccc} 
Paramètres & $\begin{array}{c}\text { Soja } \\
\text { brut }\end{array}$ & $\begin{array}{c}\text { Soja } \\
\text { cuit }\end{array}$ & $\begin{array}{c}\text { Soja } \\
\text { grillé }\end{array}$ \\
\hline Matière sèche (\% du brut) & 89,8 & 93,76 & 96,15 \\
Protéines brutes (\% MS) & 40,36 & 40,49 & 38,16 \\
Matières grasses (\% MS) & 11,8 & 13,99 & 16,49 \\
Cendres (\% MS) & 7,29 & 6,23 & 6,69 \\
Phosphore (\% MS) & 0,54 & 0,74 & 0,57 \\
NDF (\% MS) & 40,69 & 40,93 & 58,19 \\
ADF (\% MS) & 27,13 & 27,79 & 30,32 \\
Cellulose brute (\% MS) & 3,76 & 3,12 & 2,29 \\
Antitrypsine (mgN/g/min) & 10 & 5 & 5 \\
Uréase (mgN/g/min) & 0,36 & 0,11 & 0,17 \\
Coût de traitement (Fcfa/kg) & - & 50,5 & 20
\end{tabular}


Les coûts de traitement ont été évalués à partir des coûts de la main d'œuvre pour la cuisson et la grillade, le séchage, la consommation d'eau et de bois. Les coûts alimentaires de production d'un kilogramme de poids vif ont été pondérés à partir des coûts calculés sur les deux phases de croissance.

Les paramètres étudiés ont été la consommation alimentaire, le gain de poids moyen quotidien, l'indice de consommation et le coût alimentaire de production d'un kilogramme de poids vif. Les résultats obtenus ont été soumis à l'analyse de variance et la séparation des moyennes a été effectuée par la méthode de Newman et Keuls (4).

\section{RESULTATS ET DISCUSSION}

Les caractéristiques chimiques analysées des rations expérimentales et des graines de soja brutes, cuites et grillées (tableaux I et III) ont montré des valeurs élevées des constituants pariétaux et une faible teneur en matières grasses des graines par rapport aux valeurs classiques. Ces résultats seraient dus à un artefact du dosage, en particulier pour la teneur en NDF du soja grillé. Les facteurs antitrypsines et l'uréase sont passés respectivement de 10 et de $0,36 \mathrm{mgN} / \mathrm{g}$ de matières azotées dans le soja brut à 5 et $0,11 \mathrm{mgN} / \mathrm{g}$ dans le soja cuit et à 5 et $0,17 \mathrm{mgN} / \mathrm{g}$ dans le soja grillé. Ces valeurs ont été comparables à celles rapportées par Sio (13) et Tinga (14) sur les graines de soja brutes et cuites pendant 60 min et ont été conformes aux normes de qualité nutritionnelle proposées par Larbier et Leclercq (9).

Les performances zootechniques des animaux (tableau IV) ont montré en phase de croissance une augmentation non significative ( $p>0,05$ ) de la consommation alimentaire de la ration du soja cuit qui s'est traduite par un gain pondéral et une efficacité alimentaire significativement $(\mathrm{P}<0,05)$ supérieurs aux rations des tourteaux de soja et de coton. En phase de finition, une chute de performance a été enregistrée dans la ration du soja cuit à cause d'un animal malade pendant deux semaines. Cette maladie pouvait avoir été causée par l'ingestion de graines de soja moisies et mal séchées qui a entraîné une diminution du gain pondéral et une augmentation de l'indice de consommation du soja cuit par rapport au soja grillé.

La synthèse des résultats a montré que le gain pondéral et l'indice de consommation obtenus à partir des rations des graines cuites et grillées ont été significativement supérieurs $(\mathrm{p}<0,05)$ à ceux de la ration du tourteau de coton, mais ces résultats n'ont pas montré de différence significative $(\mathrm{p}>0,05)$ par rapport au tourteau de soja. Une comparaison des deux méthodes de traitement n'a présenté aucune différence significative sur les performances zootechniques des animaux bien que, numériquement, l'indice de consommation du soja cuit ait été meilleur.

L'indice de consommation de la ration du soja grillé riche en énergie a été supérieur numériquement à celui de la ration du tourteau de soja, pourtant il est établi que l'augmentation de la concentration énergétique d'une ration améliore son indice de consommation. Ceci pourrait s'expliquer par une faible utilisation digestive des nutriments de la ration du soja grillé.

Les gains de poids moyens quotidiens de 610 et $680 \mathrm{~g}$ des rations du soja grillé et cuit ont été comparables au gain moyen quotidien de 658 g (10) obtenus chez des porcs nourris au soja extrudé, et supérieurs aux gains moyens quotidiens de 490 et $470 \mathrm{~g}$ obtenus chez les porcs recevant 20 et 25 p. 100 du soja cuit pendant une heure à la température de $90{ }^{\circ} \mathrm{C}(1)$.

Une observation de deux carcasses de porc par traitement n'a pas montré de différences évidentes quant à la qualité de la viande produite. Les coûts alimentaires pondérés de production d'un kilogramme de poids vif sur les deux phases de croissance, bien qu'ils

\section{Tableau IV}

Performances des porcs nourris à base des tourteaux de soja, de coton et des graines de soja cuites ou grillées

\begin{tabular}{|c|c|c|c|c|c|c|}
\hline Période & Paramètres & TS & TC & SC & SG & ESM \\
\hline $\begin{array}{l}\text { Phase de croissance } \\
(70 \mathrm{j})\end{array}$ & $\begin{array}{l}\text { Nombre d'animaux } \\
\text { Poids initial moyen }(\mathrm{kg}) \\
\text { Consommation alimentaire }(\mathrm{kg} / \text { porc/j) } \\
\text { Gain moyen quotidien }(\mathrm{kg} / \text { porc/j) } \\
\text { Indice de consommation } \\
\text { Poids final }(\mathrm{kg} / \text { porc) }\end{array}$ & $\begin{array}{l}5 \\
15,3^{\mathrm{a}} \\
1,51^{\mathrm{a}} \\
0,57^{\mathrm{a}} \\
2,64^{\mathrm{b}} \\
55,2^{\mathrm{a}}\end{array}$ & $\begin{array}{l}5 \\
15,3^{\mathrm{a}} \\
1,48^{\mathrm{a}} \\
0,52^{\mathrm{a}} \\
2,81^{\mathrm{b}} \\
52,2^{\mathrm{a}}\end{array}$ & $\begin{array}{l}5 \\
15,5^{\mathrm{a}} \\
1,57^{\mathrm{a}} \\
0,67^{\mathrm{b}} \\
2,33^{\mathrm{a}} \\
62,8^{\mathrm{b}}\end{array}$ & $\begin{array}{l}5 \\
15,4^{\mathrm{a}} \\
1,44^{\mathrm{a}} \\
0,56^{\mathrm{a}} \\
2,53^{\mathrm{ab}} \\
55,1^{\mathrm{a}}\end{array}$ & $\begin{array}{l}1,67 \mathrm{~ns} \\
0,05 \mathrm{~ns} \\
0,04 * \\
0,14^{*} \\
3,05 *\end{array}$ \\
\hline $\begin{array}{l}\text { Phase de finition } \\
(28 \mathrm{j})\end{array}$ & $\begin{array}{l}\text { Consommation alimentaire }(\mathrm{kg} / \text { porc/j) } \\
\text { Gain moyen quotidien }(\mathrm{kg} / \text { porc/j) } \\
\text { Indice de consommation } \\
\text { Poids final moyen }(\mathrm{kg} / \text { porc/j) }\end{array}$ & $\begin{array}{r}2,08^{\mathrm{a}} \\
0,78^{\mathrm{a}} \\
2,67^{\mathrm{a}} \\
77,1^{\mathrm{a}}\end{array}$ & $\begin{array}{l}2,03^{\mathrm{a}} \\
0,55 \mathrm{c} \\
3,69^{\mathrm{b}} \\
67,6^{\mathrm{b}}\end{array}$ & $\begin{array}{l}2,19^{a} \\
0,67^{b} \\
3,26^{a b} \\
81,7^{a}\end{array}$ & $\begin{array}{c}2,13^{\mathrm{a}} \\
0,72^{\mathrm{ab}} \\
2,95^{\mathrm{a}} \\
75,3^{\mathrm{ab}}\end{array}$ & $\begin{array}{l}0,08 \mathrm{~ns} \\
0,08^{*} \\
0,33^{*} \\
4,46^{*}\end{array}$ \\
\hline $\begin{array}{l}\text { Période totale } \\
(98 \mathrm{j})\end{array}$ & $\begin{array}{l}\text { Poids initial moyen }(\mathrm{kg}) \\
\text { Consommation alimentaire }(\mathrm{kg} / \text { porc/j) } \\
\text { Gain moyen quotidien }(\mathrm{kg} / \text { porc/j) } \\
\text { Indice de consommation } \\
\text { Poids final }(\mathrm{kg} / \text { porc) }\end{array}$ & $\begin{array}{c}15,3^{\mathrm{a}} \\
1,79^{\mathrm{a}} \\
0,63^{\mathrm{a}} \\
2,84^{\mathrm{a}} \\
77,1^{\mathrm{a}}\end{array}$ & $\begin{array}{l}15,3^{\mathrm{a}} \\
1,75^{\mathrm{a}} \\
0,53^{\mathrm{b}} \\
3,30^{\mathrm{b}} \\
67,6^{\mathrm{b}}\end{array}$ & $\begin{array}{l}15,5^{\mathrm{a}} \\
1,88^{\mathrm{a}} \\
0,68^{\mathrm{a}} \\
2,76^{\mathrm{a}} \\
81,7^{\mathrm{a}}\end{array}$ & $\begin{array}{r}15,4^{\mathrm{a}} \\
1,78^{\mathrm{a}} \\
0,61^{\mathrm{a}} \\
2,91^{\mathrm{a}} \\
75,3^{\mathrm{ab}}\end{array}$ & $\begin{array}{c}167 \mathrm{~ns} \\
0,05 \mathrm{~ns} \\
0,04^{*} \\
0,15^{*} \\
4,46^{*}\end{array}$ \\
\hline & Coût alimentaire final/kg poids vif (Fcfa) & 475,6 & 457,4 & 461,9 & 455,7 & $33,28 \mathrm{~ns}$ \\
\hline $\begin{array}{l}\text { TS : tourteaux de soja } \\
\text { TC : tourteaux de coton } \\
\text { SC : graines de soja cuites } \\
\text { SG : graines de soja grillées } \\
\text { ESM : erreur standard autour } \\
\text { * Significatif }(\mathrm{p}<0,05) ; \text { ns : } \\
\text { a, b Les moyennes d'une mêm }\end{array}$ & $\begin{array}{l}\text { de la moyenne } \\
\text { non significatif } \\
\text { e ligne, affectées de la même lettre, ne }\end{array}$ & & & & & \\
\hline
\end{tabular}


n'aient pas été significativement différents, ont montré une légère baisse numérique pour le traitement par grillade $(462 \mathrm{Fcfa} / \mathrm{kg}$ pour le soja cuit contre $456 \mathrm{Fcfa} / \mathrm{kg}$ pour le soja grillé).

\section{- CONCLUSION}

Au regard des résultats obtenus, les traitements des graines de soja par la cuisson et par la grillade ne présentent pas de différences significatives sur les performances des animaux. Le traitement par grillade apparait ainsi comme une méthode économique aussi efficace que le traitement par la chaleur humide des graines de soja.

\section{Remerciements}

Ce travail a été réalisé avec l'appui financier de l'Institut de recherche agricole pour le développement (Irad) à travers le projet Banque africaine de développement (BAD). Les auteurs tiennent à remercier M. André Zoyim pour le suivi des animaux et la prise des données.

BIBLIOGRAPHIE

1. AGBEDE G., DJOUKAM J., MOGAVERO J.P., SALEY P., 1988 Détermination du taux optimal des graines de soja dans les régimes pour porcs. Tropicultura, 6 : 99-106.

2. AOAC, 1984. Official methods of analysis, 14th Edn. Washington DC, USA, Association of Official Analytical Chemists.

3. COMBS G.E, CONNESS R.G., BERRY T.H, WALLACE H.D, 1967. Effect of raw and heated soybeans on gain, nutrient digestibility, plasma amino acids and others blood constituants of growing swine. J. Amin Sci., 26: 1067-1071.

\section{Summary}

Meffeja F., Dongmo T., Njifutie N., Djoukam J., Fotso J.M. Comparative Effect of Diets Containing Cooked and Roasted Soybeans on Growth Performances of Growing Finishing Pigs

An experiment was conducted to evaluate the efficacy of heat treatment by roasting (dry heat) and cooking (moist heat) of soybeans on the performances of grower-finisher pigs. Twenty hybrid male piglets, averaging $15.4 \pm 0.8 \mathrm{~kg}$ initial body weights were allotted to four experimental diets (five replicates per treatment) based on soybean meal, cotton seed cake, cooked or roasted soybeans over a 98-day period. The rations containing the commonly used soybean meal and cotton seed cake were used as control diets. Results showed that the weight gain and feed conversion ratio obtained with diets containing roasted and cooked soybeans were significantly $(P<0.05)$ higher than those obtained with the cotton seed cake diet. However, they showed no significant difference $(p>0.05)$ compared to the soybean meal diet. Comparison of the two methods of heat treatment showed no significant difference on animal performances. Feed costs to produce one kilogram live weight, calculated as mean costs for both growth phases, although not significant between treatments, showed a slightly lower value in roasted soybean diets. These results show that the roasting method can be used with the same advantages as the cooking method.

Key words: Swine - Piglet - Soybean meal - Seed - Cooking Roasting - Growth - Cameroon.
4. DAGNELIE P., 1986. Théorie et méthodes statistiques. Applications agronomiques. Gembloux, Belgique, Presses agronomiques, 464 p.

5. DONGMO T., 1984. Utilisation des graines de soja cuites dans l'alimentation des poulets de chair. Mémoire de fin d'étude, Ensa, Yaoundé, Cameroun, $60 \mathrm{p}$.

6. GOERING H.K., VAN SOEST P.J., 1970. Forage fibre analyses (apparatus, reagent, procedures and some applications). Washington, DC, USA, USDA. (Agricultural Handbook No 379)

7. GOGNAN U., YARON A., BERK Z., ZIMMERMAN G., 1968. Effect of processing conditions on nutritive value of isolated soybean proteins. J. Agric. Food Chem., 16: 196-198.

8. KOLHMER R.H., 1993. Soybean meal and full-fat soybeans: ingredient purchasing decisions. Feed Manage., 44: 33-36.

9. LARBIER M., LECLERCQ B., 1992. Nutrition et alimentation des volailles. Paris, France, Inra, 355 p.

10. OCHETIM S., NICHOLSON H.H., 1981. Soybeans in diet of growing pigs. East Afr. For. J., 43: 298-304.

11. PUGLIESE P.L., 1984. les graines de légumineuses d'origine tropicale en alimentation animale. Maisons-Alfort, France, lemvt, 186 p.

12. POND W.G., MANER J.H., 1974. Swine production in temperate and tropical environments. San Francisco, CA, USA, WH Freeman and Company, $646 \mathrm{p}$.

13. SIO, 1983. Résultats d'analyses chimiques des graines de soja du Cameroun. Arras, France, Société industrielle des oléagineux.

14. TINGA J., 1986. Utilisation des graines de soja cuites et broyées dans l'alimentation des poules pondeuses. Mémoire fin d'étude, FASA, Dschang, Cameroun, 50 p.

Reçu le 23.01.2003, accepté le 30.01.2004

\section{Resumen}

Meffeja F., Dongmo T., Njifutie N., Djoukam J., Fotso J.M. Influencia comparada de una alimentación basada en granos de soja cocidos o asados sobre los rendimientos de cerdos en crecimiento de acabado

Se llevó a cabo un estudio para evaluar la eficiencia de los tratamientos térmicos por asado (calor seco) y por cocido (calor húmedo) de los granos de soja sobre los rendimientos zootécnicos de los cerdos en crecimiento de acabado. Veinte lechones machos híbridos, de peso medio inicial de 15,4 $\pm 0,8 \mathrm{~kg}$, se repartieron en cuatro lotes (cinco repeticiones por lote) y se sometieron a raciones alimenticias basadas en tortas de soja, de algodón y de granos de soja cocidos o asados durante un periodo de 98 días. Las raciones basadas en tortas de soja y de algodón frecuentemente utilizadas sirvieron de control. Los resultados mostraron que la ganancia ponderal y el índice de consumo obtenidos a partir de las raciones de granos cocidos y asados fueron significativamente superiores $(p<0,05)$ a los de la ración de torta de algodón, pero no mostraron diferencia significativa $(p>0,05)$ con respecto a la ración de torta de soja. La comparación de los dos métodos de tratamiento no presentó ninguna diferencia significativa sobre los rendimientos zootécnicos de los animales. Los costos alimenticios de la producción de un kilogramo de peso vivo ponderado sobre las dos fases de crecimiento -, aunque no fueron significativamente diferentes mostraron una ligera disminución numérica para el tratamiento por asado. Esto permite afirmar que este método de tratamiento puede ser utilizado con las mismas ventajas que el tratamiento por calor húmedo.

Palabras clave: Cerdo - Lechón - Torta de soja - Semilla Cocción - tostado - Crecimiento - Camerún. 